\title{
Acupuncture methods for functional constipation: protocol for a systematic review and network meta-analysis
}

\author{
Junpeng Yao ${ }^{1 \#}$, Liping Chen ${ }^{1 \#}$, Siyuan Zhou ${ }^{1}$, Yuqing Yang ${ }^{1}$, Lu Wang $^{1}$, Xiangyun Yan $^{1}$, Lin Zhang ${ }^{2}$, \\ Ying $\mathrm{Li}^{3 \wedge}$ \\ ${ }^{1}$ Acupuncture and Tuina School, Chengdu University of Traditional Chinese Medicine, Chengdu, China; ${ }^{2}$ Department of General Practice, West \\ China Hospital of Sichuan University, Chengdu, China; ${ }^{3}$ Graduate School, Chengdu University of Traditional Chinese Medicine, Chengdu, China \\ \#These authors contributed equally to this work. \\ Correspondence to: Prof. Ying Li. Graduate School, Chengdu University of Traditional Chinese Medicine, Chengdu 610075, China. \\ Email: liying@cdutcm.edu.cn.
}

Background Functional constipation (FC) is one of the most prevalent functional gastrointestinal
disorders, with the most significant negative impact on health-related quality of life. Despite that multiple
systematic reviews and clinical trials have suggested that acupuncture could be effective for FC treatment,
the comparative effectiveness among various acupuncture approaches has remained unknown. This protocol
proposed a plan to assess and rank the effectiveness and safety of different acupuncture methods for patients
with FC.
Methods: We will search 8 bibliographic databases from their inception to 30 June 2021, including
the Cochrane Central Register of Controlled Trials, MEDLINE (via PubMed), EMBASE, Allied and
Complementary Medicine Database, the Chinese Biomedical Literature Database (CBM), Wanfang
Database, VIP Database, and China National Knowledge Infrastructure (CNKI). Randomized controlled
trials (RCT) examining acupuncture methods for FC will be considered. The primary outcome is a
measurement of the weekly stool frequency [including bowel movement (BM), spontaneous bowel
movement (SBM), and complete SBM]. There will be at least 2 reviewers in charge of study selection, data
extraction, risk of bias assessment. Bayesian network meta-analysis will be conducted using ADDIS (Aggregate
Data Drug Information System) V.1.16.8. Meta-regression and subgroup analyses will also be performed,
if feasible, to address the potential causes of inconsistency and heterogeneity. Furthermore, the strength
of evidence will be appraised using the Grading of Recommendations Assessment, Development, and
Evaluation (GRADE) system.

Discussion: In this study, we will provide evidence-based hierarchies for the effectiveness and safety of acupuncture therapies to manage constipated patients, assisting healthcare providers, physicians, and patients in making more informed treatment decisions.

Trial registration: This study has been registered at PROSPERO (https://www.crd.york.ac.uk/prospero/) with a registration number CRD42021227920.

Keywords: Functional constipation (FC); acupuncture therapy; network meta-analysis (NMA)

Submitted Apr 05, 2021. Accepted for publication Jun 11, 2021.

doi: 10.21037/apm-21-811

View this article at: https://dx.doi.org/10.21037/apm-21-811

\footnotetext{
^ ORCID: Junpeng Yao, 0000-0002-0808-8428; Liping Chen, 0000-0002-6063-0261; Ying Li, 0000-0001-7554-0958.
} 


\section{Introduction}

\section{Description of the condition}

Functional constipation (FC) is a common functional bowel disorder in which symptoms of difficult, infrequent, or incomplete defecation predominate (1). According to the ROME IV criteria, patients with FC should not meet irritable bowel syndrome (IBS) criteria, although abdominal pain and/or bloating may be present but are not predominant symptoms (1). It affects around 10-15\% of the worldwide population and accounts for most clinic visits for functional intestinal disorders $(2,3)$. An epidemiological survey conducted in 2020 estimated that the average prevalence of $\mathrm{FC}$ was $7.8 \%$ in 3 English-speaking countries (7.9\% in Canada, $6.9 \%$ in the United States, and $8.6 \%$ in the United Kingdom) (4). Recurrence of FC may increase the risk for colorectal cancer and cardiovascular events and negatively impact the quality of daily life (5). The effects of FC extend beyond the individual to affect society in ways such as impairing performance at work or daily activities and thus increasing the medical costs $(6,7)$.

Conventional treatments include medical therapies such as fiber, osmotic and stimulant laxatives, and selective 5-hydroxytryptamine receptor $4\left(5-\mathrm{HT}_{4}\right)$ agonists to relieve constipation when patients respond poorly to lifestyle modifications (8). However, these treatments are limited by their unsatisfactory efficacy, drug dependence, and adverse events. Accordingly, it is not surprising that nearly $50 \%$ of constipated patients seek out alternate effective, safe, and non-toxic treatments (9-11).

\section{Description of the intervention}

As an important part of nonpharmacologic therapies, acupuncture treatment has been frequently promoted for managing FC worldwide. Several randomized controlled trials (RCT) have confirmed that acupuncture could increase the frequency of bowel movements, soften stool formation, alleviate defecation straining, and improve the quality of life (12-15). Clinically, a variety of acupuncture methods are available for FC treatment, including electroacupuncture (EA), auricular acupuncture (AA), warmneedling acupuncture (WNA), fire acupuncture (FA), and moxibustion. Recently, Liu et al. found that the effectiveness of EA was non-inferior to prucalopride in relieving severe chronic constipation (15). A comparison between AA and usual care demonstrated a significant patient preference for AA (16). Meanwhile, Park et al. showed that moxibustion has a certain effect on the management of constipation (17).

In addition, there has also been a myriad of traditional meta-analyses separately verifying the therapeutic effects of various acupuncture methods for FC (18-21). However, among the distinctive types of acupuncture approaches, the most effective method has not yet been determined. This is mainly a result of there have been no head-to-head comparisons between different acupuncture methods and that pairwise meta-analyses fail to establish a hierarchy of the optimal intervention for a condition. Clinically, the apparent uncertainty has resulted in physicians experiencing confusion regarding selecting the optimal acupuncture therapy for constipated patients (22).

\section{How the intervention might work}

Mechanisms underlying the various methods of acupuncture for FC are still not very clear. Previous studies have demonstrated that acupuncture could improve gastrointestinal motility, promote contractility of colonic tissue, and facilitate gut transit in constipated animals $(23,24)$. Zhu et al. observed that EA could increase the release of serotonin in the distal colon, including 5-hydroxytryptamine and tryptophan hydroxylase (25). $\mathrm{Xu}$ et al. showed that intestinal dysbiosis contributed to constipation, and MA could rebalance the gut microbiota by decreasing the Firmicutes/Bacteroidetes ratio (26). Meanwhile, high-frequency EA stimulation may regulate activity in the enteric nervous system and regenerate the lost enteric neurons (27). Furthermore, $\mathrm{Xu}$ et al. found that moxibustion could increase gastrointestinal movement by modulating the level of serum motilin and gastrin (28).

\section{Why it is important to perform this review}

In recent years, network meta-analyses have provided precisely effective strategies for assessing multiple competing interventions (29). By incorporating results from both indirect and direct evidence, network meta-analyses allow estimation of intervention effects where head-tohead comparisons are scarce and further obtain the efficacy ranking of various methods (30). To date, no published network meta-analysis has been completed to identify the optimal acupuncture method for FC treatment. Thus, we saw an opportunity to conceive a comprehensive network meta-analysis to answer the following pivotal questions: (I) which acupuncture method is the most effective for FC and specific categories of patients with FC? (II) What is the 
optimal acupuncture regimen to improve gastrointestinal symptoms and quality of life in patients with FC? We present the following article in compliance with the PRISMA-P reporting checklist (available at https://dx.doi. org/10.21037/apm-21-811).

\section{Objectives}

This network meta-analysis aims to compare the effectiveness and safety of all available acupuncture methods in the management of patients with FC.

\section{Methods}

The proposed systematic review was registered on PROSPERO (CRD42021227920) and is available on the PROSPERO.com website (https://www.crd.york.ac.uk/ prospero/). The protocol is reported in accordance with the PRISMA-Protocols (PRISMA-P) statement and its extension for Network Meta-analyses (31).

\section{Criteria for including studies in this review}

\section{Types of studies}

The review will include all relevant RCTs, without any language or regional restrictions. Only completed studies will be included. If the study was designed as a randomized crossover trial, only the first-phase results will be included. However, ongoing studies, animal studies, quasi-RCTs, case reports and expert experience should be excluded.

\section{Types of participants}

Adult patients ( $\geq 18$ years) who have been diagnosed with FC, irrespective of their ethnic background, gender, or initial severity of the condition, will be included. Pediatric patients will be excluded from this NMA. Diagnosis will be made according to the Rome IV/III/II criteria or other accepted diagnostic standards (1,2). Patients with constipation co-presenting with irritable bowel syndrome or secondary constipation (drug-induced, postoperative, or attributable to other diseases) should be excluded.

\section{Types of interventions}

Studies reporting on the use of acupuncture therapy as a primary intervention will be included. We will define the acupuncture approaches as needle stimulation of acupoints, including MA, EA, auricular acupuncture/acupressure, WNA, FA, and moxibustion. Therefore, acupoint catgut embedding, cupping, acupoint injection, blood-letting therapy, and herbal medicine should be excluded due to their use of related drugs.

\section{Types of control groups}

Treatment methods of non-acupuncture and nonmoxibustion will form the basis of the comparison groups, such as sham acupuncture (invasive/non-invasive), no treatment/waiting list/lifestyle modifications, and recommended drugs (fiber, stimulant and osmotic laxatives, and selective $5-\mathrm{HT}_{4}$ agonists). Studies comparing different manipulations of acupuncture or alternate selections of acupoints will be excluded.

\section{Types of outcome measures}

Trials that cover 1 or more of the below-mentioned outcomes will be included. Otherwise, the study will be excluded.

\section{Primary outcomes}

The primary objective of our review will be the investigation of different acupuncture methods in the improvement of the frequency of bowel movements. Hence, the primary outcomes will be the weekly stool frequency [including bowel movement (BM), spontaneous bowel movement (SBM), and complete SBM] at the end of treatment and follow-up. An SBM is described as a bowel movement that occurred without assistance from drugs or other techniques during the past $24 \mathrm{~h}(13,15)$. A complete SBM is described as an SBM with a feeling of complete evacuation $(13,15)$.

\section{Secondary outcomes}

The secondary outcomes will include: (I) stool consistency assessed with Bristol Stool Form Scale (BSFS) score. The BSFS score is a 7-point scale, with scores of 1-2 indicating constipation, 3-5 indicating normal stool, and 6-7 indicating diarrhea; (II) responder rate, a responder was defined as no longer fulfilling the Rome criteria for FC (32); (III) the Patient Assessment of Constipation Quality of Life questionnaire; and (IV) the incidence and severity of adverse events (including hematomas, dizziness, or local pain) will also be measured for safety assessment.

\section{Search methods for identification of studies}

\section{Electronics searches}

This study will search, both electronically and manually, 
Table 1 Search strategy used in PubMed

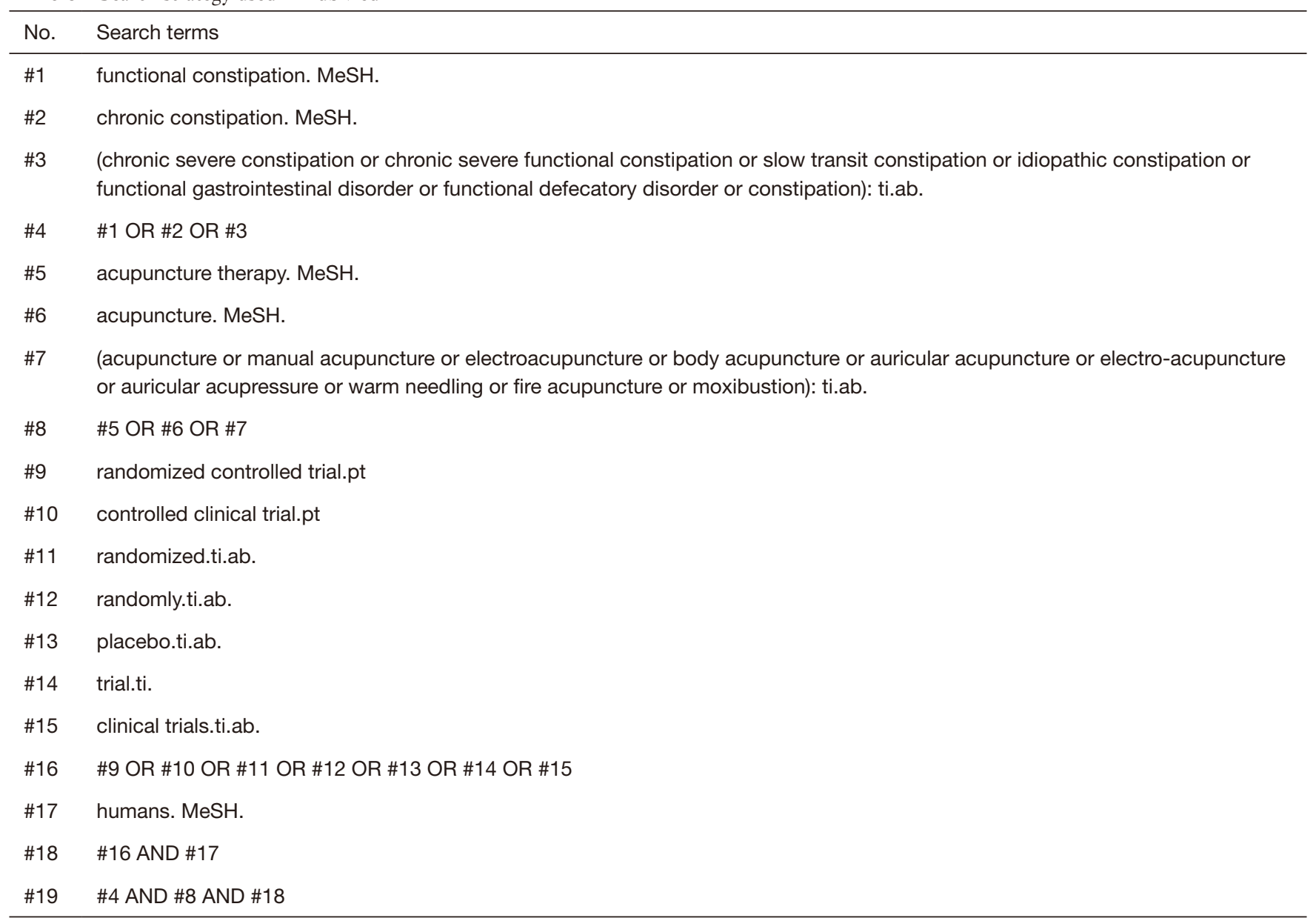

for all RCTs involving acupuncture methods for FC, regardless of publication status. The following bibliographic databases will be searched up to 30 June 2021: Cochrane Central Register of Controlled Trials, MEDLINE (via PubMed), EMBASE, Allied and Complementary Medicine Database, the Chinese Biomedical Literature Database (CBM), Wanfang Data, VIP Database, and China National Knowledge Infrastructure (CNKI). The search strategy will be structured by Medical Subject Headings (MeSH) and free-text terms, such as functional constipation OR constipation AND acupuncture therapy OR acupuncture OR electroacupuncture OR acupuncture-moxibustion AND randomized. The detailed search strategy for PubMed is as described in Table 1. Based on the characteristics of each database, this search strategy will be adjusted to individual database constraints.

\section{Searching other resources}

Any relevant ongoing or unpublished clinical study will be obtained from the World Health Organization (WHO) International Clinical Trials Registry Platform, the National Institutes of Health (NIH) clinical registry, and the Chinese clinical registry. The reference lists of the selected studies and published systematic reviews will be screened for additional literature. Moreover, a manual search will be performed for grey literature, including conference proceedings. Experts in the field will also be consulted to obtain potential studies and the most recent clinical data not available by the previously mentioned methods.

\section{Selection of studies}

During the study selection process, search results will be 

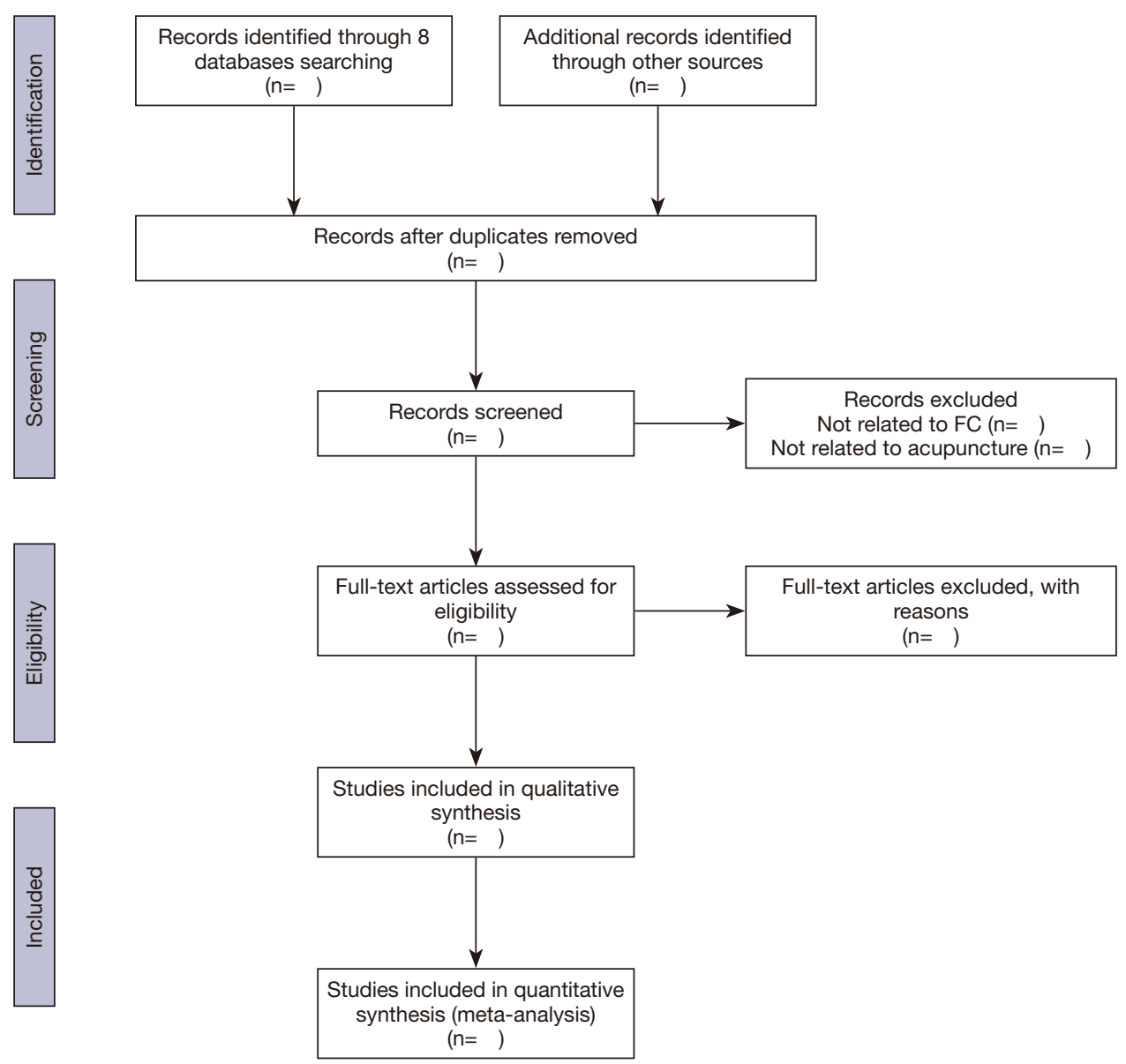

Figure 1 The PRISMA flow diagram of studies identified.

imported from the original databases to Endnote $\mathrm{X} 9$, and the software will filter repetitive studies. Then, 2 reviewers (JY and LC) will independently screen all the retrieved records by reading the title, abstract, and keywords to determine which studies meet the inclusion criteria. The full text will be obtained and checked independently by the 2 reviewers (JY and LC), where a study is potentially eligible. Notably, studies excluded after reading the full text will be recorded and explained. The detailed process of selection is shown in Figure 1. Any disagreement will be resolved by consensus, and further conflicts will be arbitrated by a third reviewer (LZ).

\section{Data extraction and management}

The following data will be independently extracted from the eligible studies using a self-designed data acquisition form by the 2 reviewers (SZ and YY): (I) details of the study (publication year, first author, nationality, study design); (II) patient demographics (sample size, age, gender, ethnicity, diagnosis, constipation duration); (III) intervention and comparator (types of acupuncture, selected acupoints, treatment duration/frequency/session, follow-up period); (IV) outcomes [types of outcomes, definition of outcome, time points for each assessment, related statistics such as mean \pm standard deviation (SD) and $\mathrm{P}$ value]; (V) adverse reactions; (VI) main conclusion and funding sources. If the original trials had more than 2 groups, the data would be extracted separately. When the mean and SD are not offered directly in the study, we will attempt to obtain them through a calculation based on our previous study or by contacting the corresponding authors (19). Any discrepancy noticed during the process of data extraction will be resolved through discussion or suggestions from a third reviewer (YL). 


\section{Quality assessment}

For quality assessment, 2 reviewers (LW and XY) will use the Cochrane Risk of Bias tool to assess the risk of bias based on the following 6 aspects: potential selection bias (random sequence generation and concealment of allocation), performance bias (blinding of investigators and participants), detection bias (blinding of outcome assessors), attrition bias (incomplete outcome data), reporting bias (selective reporting), and other possible sources of bias (funding bias) (33). Each trial will be judged as "low", "unclear", or "high" risk of bias. If inconsistent results appear, a final decision will be made by the third reviewer (LZ).

The 2 reviewers (JY and LC) will independently appraise the strength of the evidence using the Grading of Recommendations Assessment, Development, and Evaluation (GRADE) system (34). Rating the quality of evidence begins by assigning a designation of "high-quality", and this designation may be reduced to "moderate quality", "low quality", or "very low quality" based on variables such as the risk of bias, imprecision, heterogeneity or inconsistency, and/or publication bias. Any discrepancy will be arbitrated by a third reviewer (YL).

\section{Dealing with missing data}

Where unclear or missing data are present, an effort will be made to contact the first or corresponding author to request additional information. For the continuous outcomes, when both intention-to-treat analyses and per-protocol are reported, we will prioritize the former. If the authors do not respond, we will assume the missing value with the last-observation-carried-forward imputation method (35). If continuous variables are not offered as mean and SD, we will calculate the missing data by converting standard errors, $95 \%$ confidence interval (CI), or $\mathrm{P}$ values into SD. For the categorical variables, patients with missing data will be assumed not to have changed from baseline (36). Moreover, the potential impact of missing data will be tested by conducting sensitivity analyses and disclosed in the final publication.

\section{Assessment of similarity and consistency}

To obtain a credible and valid result, the judgment of similarity and consistency assumption will be necessary. Comparison of the distribution of clinical and methodological variables across studies will be applied to address similarity, including patient characteristics (age and severity of the disease), trial designs (blinding and risk of bias), and interventions (types, sessions, intensity, and duration).

The assumption of consistency will be assessed using both a local and global approach. The node-splitting approach will assess the local consistency of direct and indirect evidence, and the $\mathrm{P}$ value will be calculated (37). If the node shows a $\mathrm{P}$ value greater than 0.05 , the implication will be that no significant statistical difference existed; on the contrary, the comparison of direct and indirect evidence will be regarded as inconsistent (38). Meanwhile, the design-by-treatment interaction model and chi-squared test will be used to assess inconsistency throughout the entire network (39).

\section{Statistical analysis}

\section{Pairwise meta-analysis}

Pairwise meta-analysis will be performed using the software RevMan version 5.4 (Review Manager, The Cochrane Collaboration, 2020) to compare treatments with direct evidence. Post-treatment values will be used to calculate the effect sizes. The heterogeneity within each pairwise comparison will be assessed with the $\mathrm{Q}$ test and $\mathrm{I}^{2}$ value. If the $\mathrm{I}^{2}$ value is below $50 \%$, a fixed-effects model will be used; otherwise, a random-effects model will be employed. For continuous variables, the mean difference (MD) or standardized mean difference (SMD) with their 95\% CIs will be measured as the effect size, whereas the categorical variables will be presented as relative risk (RR) or odds ratio (OR) alongside their 95\% CIs.

\section{Network meta-analysis}

We will use Aggregate Data Drug Information System (ADDIS) V.1.16.8 software (Drugis, Groningen, NL) to conduct the network meta-analysis $(38,40)$. A Bayesian network meta-analysis will be performed using a random effects model for integrating the direct and indirect evidence with the Markov Chain Monte Carlo method. The parameters will be set at 4 chains for simulation, while each chain will have 50,000 simulation iterations, and the first 20,000 simulations will be discarded to eliminate the effect of the initial value. Model convergence will be assessed by visual inspection of the trace plots and after considering the Gelman-Rubin statistic. Meanwhile, STATA V.15.0 software (StataCorp., College Station, TX, USA) will be 
used to generate the network diagram and compare each outcome (38). For each specific outcome, we will sequence the effects of different acupuncture methods to determine the most effective surface under the cumulative ranking curve and mean ranks alongside their $95 \%$ CI.

\section{Assessment of heterogeneity}

Statistical heterogeneity among studies will be assessed using the $\mathrm{Q}$ test and $\mathrm{I}^{2}$ statistics. According to the Cochrane Handbook, the thresholds for the interpretation of the $\mathrm{I}^{2}$ statistics are as follows: $0-40 \%$, might not be important; 30-60\%, may reflect moderate heterogeneity; $50-90 \%$, may indicate substantial heterogeneity; $75-100 \%$, represents considerable heterogeneity (41). After statistical heterogeneity is established, the researchers will search for possible sources from the clinical and methodological perspective then perform subgroup or sensitivity analysis to detect the possible causes of heterogeneity.

\section{Meta-regression, subgroup analysis and sensitivity analysis}

Meta-regression and subgroup analysis will also be performed, whenever feasible, to detect the possible sources of inconsistency and heterogeneity. Meta-regression will be conducted with the following covariates: (I) initial severity of the disease; (II) sample size; (III) average age; and (IV) the duration, frequency, and course of acupuncture methods. Subgroup analyses will be employed based on the different comparators and various forms of acupuncture methods.

Multiple sensitivity analyses for the analyzed outcomes will be performed to assess the findings' robustness and examine if any particular study accounted for a large proportion of heterogeneity. The analyses will be based on different statistical models and qualities of methodology. The meta-analysis will be repeated, and more inferior quality studies will be excluded.

\section{Assessment of publication bias}

A comparison-adjusted funnel plot will be used to inspect reported bias and small-scale effects visually. If a sufficient number of included studies (more than 10 trials) are available, the funnel plot will be used to assess the reported bias (19). If the funnel plot is found to be asymmetrical, Egger's regression test will be used to analyze the causes of asymmetry. All eligible trials, regardless of the quality of their methodologies, will be included.

\section{Assessment of reviewer agreement}

To achieve perfect consistency (at least $80 \%$ ) between the 2 reviewers who conducted the study selection, data extraction, and quality assessment, a pilot study will be conducted before these processes are implemented. A Kappa index will be utilized during the pilot study to assess the inter-rater agreement between the reviewers. We interpreted thresholds for the Kappa index as greater than 0.75 , reflecting perfect agreement, $0.4-0.75$ indicating moderate agreement, and a Kappa index less than 0.4 indicating a low level of consistency (42).

\section{Ethics and informed consents}

Given that the data is not individualized, formal ethical approval is not required. All analyzed trials will have followed the Declaration of Helsinki (as revised in 2013) and current ethical standards. Additionally, the findings from this study will be published in a peer-reviewed journal. We also plan to disseminate this study, including pilot results, through the conference presentations.

\section{Discussion}

Multiple acupuncture therapies have been reported as effective for FC; however, there is high variability among clinicians in using these acupuncture methods. Our previous standard pairwise meta-analyses that combined evidence from 30 RCTs indicated that acupuncture was superior to laxatives in terms of stool frequency and improving the quality of daily life (19). Based on the previous work, this Bayesian network meta-analysis will compare the effectiveness of all available acupuncture therapies and rank the probability of acupuncture methods concerning each domain of FC. We expect that our findings will help healthcare providers, physicians, and patients to make more precise treatment decisions. Moreover, the results of this study will also apply to informing evidence-based guidelines for FC treatment. Any deviations from the procedures described in this protocol will be updated in PROSPERO and, therefore, report the rationale for and the date of these amendments in the final report.

\section{Strengths and limitations}

This study will also have several strengths and limitations. Firstly, this will be the first Bayesian network meta-analysis 
attempted to establish evidence-based hierarchies for the effectiveness and safety of all acupuncture therapies for the management of constipated patients. The results of this study will assist healthcare providers, physicians, and patients in making more accurate treatment decisions. Secondly, 2 or more researchers will independently conduct the study selection, data extraction, and quality assessment to ensure that all relevant studies will be collected without personal bias. Regarding limitations, our proposed methodology will focus on acupuncture methods, and less emphasis will be placed on the analysis of specific details concerning acupuncture application or the selection of acupoints.

\section{Acknowledgments}

We would like to thank J. Jones and J. Chapnick for their kind help in polishing our paper.

Funding: This work was supported by grants from the National Natural Science Foundation of China (NO. 82074554 and 81774430) and the Foundation of the Science and Technology Department of Sichuan Province, China (NO. 2021YJ0197).

\section{Footnote}

Reporting Checklist: The authors have completed the PRISMA-P reporting checklist. Available at https://dx.doi. org/10.21037/apm-21-811

Peer Review File: Available at https://dx.doi.org/10.21037/ apm-21-811

Conflicts of Interest: All authors have completed the ICMJE uniform disclosure form (available at https://dx.doi. org/10.21037/apm-21-811). The authors have no conflicts of interest to declare.

Ethical Statement: The authors are accountable for all aspects of the work in ensuring that questions related to the accuracy or integrity of any part of the work are appropriately investigated and resolved. Given that the data is not individualized, formal ethical approval is not required. All analyzed trials will have followed the Declaration of Helsinki (as revised in 2013) and current ethical standards. This study has been registered at PROSPERO (https:// www.crd.york.ac.uk/prospero/) with a registration number CRD42021227920.
Open Access Statement: This is an Open Access article distributed in accordance with the Creative Commons Attribution-NonCommercial-NoDerivs 4.0 International License (CC BY-NC-ND 4.0), which permits the noncommercial replication and distribution of the article with the strict proviso that no changes or edits are made and the original work is properly cited (including links to both the formal publication through the relevant DOI and the license). See: https://creativecommons.org/licenses/by-nc-nd/4.0/.

\section{References}

1. Mearin F, Lacy BE, Chang L, et al. Bowel Disorders. Gastroenterology 2016;S0016-5085(16)00222-5. [Epub ahead of print]. doi:10.1053/j.gastro.2016.02.031

2. Vriesman MH, Koppen IJN, Camilleri M, et al. Management of functional constipation in children and adults. Nat Rev Gastroenterol Hepatol 2020;17:21-39.

3. Peery AF, Crockett SD, Barritt AS, et al. Burden of Gastrointestinal, Liver, and Pancreatic Diseases in the United States. Gastroenterology 2015;149:1731-1741.e3.

4. Palsson OS, Whitehead W, Törnblom H, et al. Prevalence of Rome IV Functional Bowel Disorders Among Adults in the United States, Canada, and the United Kingdom. Gastroenterology 2020;158:1262-1273.e3.

5. Sumida K, Molnar MZ, Potukuchi PK, et al. Constipation and risk of death and cardiovascular events. Atherosclerosis 2019;281:114-20.

6. Sethi S, Mikami S, Leclair J, et al. Inpatient burden of constipation in the United States: an analysis of national trends in the United States from 1997 to 2010. Am J Gastroenterol 2014;109:250-6.

7. Mohaghegh Shalmani H, Soori H, Khoshkrood Mansoori $\mathrm{B}$, et al. Direct and indirect medical costs of functional constipation: a population-based study. Int J Colorectal Dis 2011;26:515-22.

8. Bharucha AE, Lacy BE. Mechanisms, Evaluation, and Management of Chronic Constipation. Gastroenterology 2020;158:1232-1249.e3.

9. Lahner E, Bellentani S, Bastiani RD, et al. A survey of pharmacological and nonpharmacological treatment of functional gastrointestinal disorders. United European Gastroenterol J 2013;1:385-93.

10. Basilisco G; Italian Society of Neurogastroenterology Motility (SINGEM) Study Group. Patient dissatisfaction with medical therapy for chronic constipation or irritable bowel syndrome with constipation: analysis of $\mathrm{N}$-of-1 prospective trials in 81 patients. Aliment Pharmacol Ther 
2020;51:629-36.

11. Johanson JF, Kralstein J. Chronic constipation: a survey of the patient perspective. Aliment Pharmacol Ther 2007;25:599-608.

12. Tjen-A-Looi SC, Fu LW. Sustained effects of acupuncture in treatment of chronic constipation. Ann Palliat Med 2017;6:S124-7.

13. Liu Z, Yan S, Wu J, et al. Acupuncture for Chronic Severe Functional Constipation: A Randomized Trial. Ann Intern Med 2016;165:761-9.

14. Lee HY, Kwon OJ, Kim JE, et al. Efficacy and safety of acupuncture for functional constipation: a randomised, sham-controlled pilot trial. BMC Complement Altern Med 2018;18:186.

15. Liu B, Wu J, Yan S, et al. Electroacupuncture vs Prucalopride for Severe Chronic Constipation: A Multicenter, Randomized, Controlled, Noninferiority Trial. Am J Gastroenterol 2021;116:1024-35.

16. Li MK, Lee TF, Suen KP. Complementary effects of auricular acupressure in relieving constipation symptoms and promoting disease-specific health-related quality of life: A randomized placebo-controlled trial. Complement Ther Med 2014;22:266-77.

17. Park JE, Sul JU, Kang K, et al. The effectiveness of moxibustion for the treatment of functional constipation: a randomized, sham-controlled, patient blinded, pilot clinical trial. BMC Complement Altern Med 2011;11:124.

18. Yao F, Zhang Y, Kuang X, et al. Effectiveness and Safety of Moxibustion on Constipation: A Systematic Review and Meta-Analysis. Evid Based Complement Alternat Med 2020;2020:8645727.

19. Wang L, Xu M, Zheng Q, et al. The Effectiveness of Acupuncture in Management of Functional Constipation: A Systematic Review and Meta-Analysis. Evid Based Complement Alternat Med 2020;2020:6137450.

20. Zhou SL, Zhang XL, Wang JH. Comparison of electroacupuncture and medical treatment for functional constipation: a systematic review and meta-analysis. Acupunct Med 2017;35:324-31.

21. Yang LH, Duan PB, Du SZ, et al. Efficacy of auriculotherapy for constipation in adults: a systematic review and meta-analysis of randomized controlled trials. J Altern Complement Med 2014;20:590-605.

22. Zheng H, Chen Q, Chen M, et al. Nonpharmacological conservative treatments for chronic functional constipation: A systematic review and network metaanalysis. Neurogastroenterol Motil 2019;31:e13441.

23. Wang X, Yang B, Yin J, et al. Electroacupuncture via chronically implanted electrodes improves gastrointestinal motility by balancing sympathovagal activities in a rat model of constipation. Am J Physiol Gastrointest Liver Physiol 2019;316:G797-805.

24. Liang C, Wang KY, Gong MR, et al. Electro-acupuncture at ST37 and ST25 induce different effects on colonic motility via the enteric nervous system by affecting excitatory and inhibitory neurons. Neurogastroenterol Motil 2018;30:e13318.

25. Zhu X, Liu Z, Qu H, et al. The effect and mechanism of electroacupuncture at LI1 1 and ST37 on constipation in a rat model. Acupunct Med 2016;34:194-200.

26. Xu M, Wang L, Guo Y, et al. Positive effects of electroacupuncture treatment on gut motility in constipated mice is related to rebalancing the gut microbiota. Evid Based Complement Alternat Med 2021;2021:6652017.

27. Du F, Liu S. Electroacupuncture with high frequency at acupoint ST-36 induces regeneration of lost enteric neurons in diabetic rats via GDNF and PI3K/AKT signal pathway. Am J Physiol Regul Integr Comp Physiol 2015;309:R109-18.

28. Xu J, Du Y, Zhang X, et al. Effects of different methods of acupuncture and moxibustion on functional constipation in rats:a comparative study. Zhongguo Zhen Jiu 2017;37:527-33.

29. Wu T, Fu C, Deng Y, et al. Acupuncture therapy for radiotherapy-induced adverse effects: a protocol for systematic review and Bayesian network meta-analysis. Ann Palliat Med 2021;10:2254-9.

30. Cipriani A, Higgins JP, Geddes JR, et al. Conceptual and technical challenges in network meta-analysis. Ann Intern Med 2013;159:130-7.

31. Shamseer L, Moher D, Clarke M, et al. Preferred reporting items for systematic review and meta-analysis protocols (PRISMA-P) 2015: elaboration and explanation. BMJ 2015;350:g7647.

32. Koppen IJN, Saps M, Lavigne JV, et al. Recommendations for pharmacological clinical trials in children with functional constipation: The Rome foundation pediatric subcommittee on clinical trials. Neurogastroenterol Motil 2018;30:e13294.

33. Sterne JAC, Savović J, Page MJ, et al. RoB 2: a revised tool for assessing risk of bias in randomised trials. BMJ 2019;366:14898.

34. Atkins D, Best D, Briss PA, et al. Grading quality of evidence and strength of recommendations. BMJ 2004;328:1490. 
35. de Goeij MC, van Diepen M, Jager KJ, et al. Multiple imputation: dealing with missing data. Nephrol Dial Transplant 2013;28:2415-20.

36. Yang M, Du T, Sun M, et al. Acupuncture for stable angina pectoris: a systematic review protocol. BMJ Open 2018;8:e019798.

37. Dias S, Welton NJ, Caldwell DM, et al. Checking consistency in mixed treatment comparison meta-analysis. Stat Med 2010;29:932-44.

38. Yin Z, Geng G, Xu G, et al. Acupuncture methods for allergic rhinitis: a systematic review and bayesian metaanalysis of randomized controlled trials. Chin Med 2020;15:109.

39. Shen Y, Jin S, Lin C, et al. Safety of different carotid artery revascularization strategies in the coronary artery bypass graft population: study protocol for a systematic review and network meta-analysis. Ann Transl Med 2020;8:1605.

40. Huang YY, Dou KX, Zhong XL, et al. Pharmacological treatment of neuropsychiatric symptoms of dementia: a network meta-analysis protocol. Ann Transl Med 2020;8:746.

41. Higgins JP, Green S. Cochrane Handbook for systematic reviews of interventions. Wiley Online Library 2008.

42. Luo YN, Zheng QH, Liu ZB, et al. Methodological and reporting quality evaluation of systematic reviews on acupuncture in women with polycystic ovarian syndrome: A systematic review. Complement Ther Clin Pract 2018;33:197-203.
Cite this article as: Yao J, Chen L, Zhou S, Yang Y, Wang L, Yan X, Zhang L, Li Y. Acupuncture methods for functional constipation: protocol for a systematic review and network meta-analysis. Ann Palliat Med 2021;10(7):8300-8309. doi: 10.21037/apm-21-811 